\title{
Application of different patella height indices in patients undergoing total knee arthroplasty
}

\author{
Bin Xu, Wei-xing Xu, Di Lu, Hong-feng Sheng, Xin-wei Xu and Wei-guo Ding ${ }^{*}$ (D)
}

\begin{abstract}
Background: One complication of total knee arthroplasty (TKA) is patella baja (PB). Patellar tendon shortening and joint line elevation are two main causes of PB. The purpose of this study was to determine the incidence of PB before and after TKA by measuring the patellar height and provide evidence for choosing a suitable index.

Methods: In total, 256 consecutive patients who underwent primary TKA were included in this study. Radiographic measurements were performed; the Insall-Salvati (IS) index, modified IS (MIS) index, Blackburne-Peel (BP) index, and Caton-Deschamps (CD) index were computed; and the incidence of PB was calculated before and after the operation. The consistency between the IS and MIS indices and between the BP and CD indices was analyzed.

Results: The preoperative incidence of true PB (TPB) and pseudo-PB (PPB) was 9.4 and $0.8 \%$, respectively. The postoperative incidence of TPB and PPB was 10.2 and 9.0\%, respectively. The consistency between the IS and MIS indices was moderate preoperatively (pre-kappa $=0.602$ ) and postoperatively (post-kappa $=0.742$ ). The consistency between the BP and CD indices was moderate preoperatively (pre-kappa $=0.742$ ) and good postoperatively (post-kappa $=0.797$ ).

Conclusion: The incidence of PB, especially PPB, increased after TKA. The CD and BP indices are of greater importance for the diagnosis of PB after TKA. The MIS index is a better choice than the IS index to measure the length of the patellar tendon. To measure the height of the joint line, the BP index is better postoperatively and the CD index is better preoperatively.
\end{abstract}

Keywords: Total knee arthroplasty, Radiographic measurement, Patella baja, Consistency test

\section{Background}

Total knee arthroplasty (TKA) is an efficient treatment for end-stage knee osteoarthropathy [1]. However, both patellar tendon shortening and joint line elevation can result in patella baja $(\mathrm{PB})[2,3]$. $\mathrm{PB}$ may cause anterior knee pain [3-6], limited range of motion (ROM) [3, 7], joint stiffness $[8,9]$, and prosthetic impact and abrasion $[9,10] . \mathrm{PB}$ is divided into true $\mathrm{PB}(\mathrm{TPB})$ and pseudo- $\mathrm{PB}$ (PPB). TPB is caused by a short patellar tendon and results in pathological values for all indices, including the Insall-Salvati (IS) index [11], modified IS (MIS) index [12], Blackburne-Peel (BP) index [13], and CatonDeschamps (CD) index [14]. The IS and MIS indices

\footnotetext{
* Correspondence: spotxu@163.com

Department of Orthopaedics, Tongde Hospital of Zhejiang Province, 234 Gu-cui Road, Hangzhou 310012, People's Republic of China
}

reflect the length of the patellar tendon, whereas the $\mathrm{BP}$ and $C D$ indices reflect the distance between the distal pole of the patella and the tibial plateau. PPB is caused by joint line elevation without a short patellar tendon and results in pathological values for the $\mathrm{CD}$ and $\mathrm{BP}$ indices, whereas the IS and MIS indices stay within the normal range.

$\mathrm{PB}$ is not an uncommon complication, and its incidence has been reported as 34,37 , and $65 \%$ in different studies [15-17]. Postsurgical PB is mainly caused by patellar tendon shortening and joint line elevation $[3,18,19]$. However, which factor plays a more important role in the development of postsurgical PB remains unknown. The consistency between the IS and MIS indices and between the $\mathrm{BP}$ and $\mathrm{CD}$ indices is also unknown. While identification of the most important risk factor for postsurgical $\mathrm{PB}$ 
is a key to the prevention of PB after TKA, evaluation of the patellar height before TKA and determination of the optimal range of the above-mentioned indices to prevent $\mathrm{PB}$ are also important. How to combine these indices to evaluate the patellar height and diagnose $\mathrm{PB}$ remains unresolved.

The present study was performed to determine the consistency between the IS and MIS indices and between the BP and CD indices before and after TKA with the overall aim of identifying a suitable index for the diagnosis of PB. Through analysis of the aforementioned indices, we also investigated whether patellar tendon shortening or joint line elevation plays a more important role in the occurrence of postoperative PB.

\section{Methods}

\section{Patient selection}

From June 2015 to November 2016 at our institution, patients undergoing primary TKA for end-stage osteoarthropathy were included in this study. The inclusion criteria were the need for primary TKA, no extensor mechanism damage, no patellar dysontogenesis or fracture, and no history of trauma or surgery. The exclusion criteria were severe deformity, preoperative $\mathrm{ROM}$ of $\leq 90^{\circ}$, varus-valgus deformity of $\geq 15^{\circ}$ [20], and severe bone defects of the femur or tibia.

\section{Operation protocol}

All operations were performed with the patient under general anesthesia or combined spinal epidural anesthesia. All total knee prostheses were posterior stabilized cemented implants (P.F.C. Sigma; DePuy Orthopaedics, Raynham, MA, USA). We performed the operations with a midline incision and medial parapatellar approach. Distal femoral osteotomy was performed based on the extramedullary alignment system according to the knee physical valgus angle, which was measured preoperatively on anteroposterior weight-bearing radiographs. Tibia preparation was performed based on the intramedullary alignment system. The patella was routinely everted during the operation, patella reshaping was performed, and no patella resurfacing was carried out.

\section{Outcome measurement}

$\mathrm{X}$-ray examinations of the knee joint in the $30^{\circ}$ flexed position were performed before and after the operation. Software (syngo Imaging; Siemens, Munich, Germany) was employed to measure the diagonal length of the patella, the length of the patellar tendon, the length of the patella articular surface, the distance from the patella articular surface to the tibial tuberosity, the perpendicular height from the plateau line of the tibia to the lower end of the patella articular surface, and the distance between the patella articular surface and anterior border of the tibial plateau in the lateral view of the knee joint. The IS, MIS, BP, and CD indices were computed (see Fig. 1). TPB was diagnosed when the IS index was $\leq 0.8$ or MIS index was $\leq 1.2$, with a BP index of $\leq 0.54$ or $\mathrm{CD}$ index of $\leq 0.60$ (see Fig. 2a). PPB was diagnosed when the IS and MIS indices were within the normal range, while the $\mathrm{BP}$ index was $\leq 0.54$ or $\mathrm{CD}$ index was $\leq 0.60$ (see Fig. 2b) [2, 11-14, 21]. The incidence of TPB and PPB were calculated both preoperatively and postoperatively. To minimize interobserver variation, two blind observers performed the measurements and calculations in the radiograph. The mean of the results from the two observers were used for the final analysis.

\section{Statistical analysis}

All data were analyzed using SPSS version 19.0 (IBM Corp., Armonk, NY, USA). The kappa test was employed to analyze the consistency between the IS and MIS indices and between the $\mathrm{BP}$ and $\mathrm{CD}$ indices. The consistency was poor when kappa $\leq 0.04$, moderate when $0.4<$ kappa $<0.75$, and good when kappa $\geq 0.75$.

\section{Results}

\section{General patient characteristics}

Among the 256 patients included in this study, 42 were men and 214 were women. Their average age was $64.56 \pm 7.98$ years, and their average body mass index was $25.77 \pm 3.56 \mathrm{~kg} / \mathrm{m}^{2}$.

\section{Incidence of PB}

Preoperatively, the incidence of TPB and PPB was 9.4\% (24 cases) and $0.8 \%$ ( 2 cases), respectively. The positive rate of the IS index was $7.8 \%$ (20 cases), MIS index was $5.9 \%$ (15 cases), BP index was $3.1 \%$ (8 cases), and CD index was $3.1 \%$ (8 cases). Postoperatively, the incidence of TPB and PPB was $10.2 \%$ (26 cases) and 9.0\% (23 cases), respectively. The positive rate of the IS index was 8.2\% (21 cases), MIS index was 9.0\% (23 cases), BP index was $15.2 \%$ (39 cases), and CD index was $14.1 \%$ (36 cases).

The consistency between the IS and MIS indices was moderate both preoperatively (pre-kappa $=0.602$ ) and postoperatively (post-kappa $=0.742$ ), but was greater postoperatively than preoperatively. The consistency between the BP and CD indices was moderate preoperatively (pre-kappa $=0.742$ ) and good postoperatively $($ post-kappa $=0.797)($ see Table 1$)$.

\section{Changes in parameters}

Compared with the preoperative value, the MIS index decreased after TKA (preoperative: $1.61 \pm 0.22$, postoperative: $1.51 \pm 0.21 ; P<0.0001$ ), reflecting patellar tendon shortening. The BP index (preoperative: $0.86 \pm 0.16$, postoperative: $0.74 \pm 0.14 ; P<0.0001$ ) and $C D$ index (preoperative: $0.93 \pm 0.15$, postoperative: $0.78 \pm 0.12 ; P<0.0001$ ) 


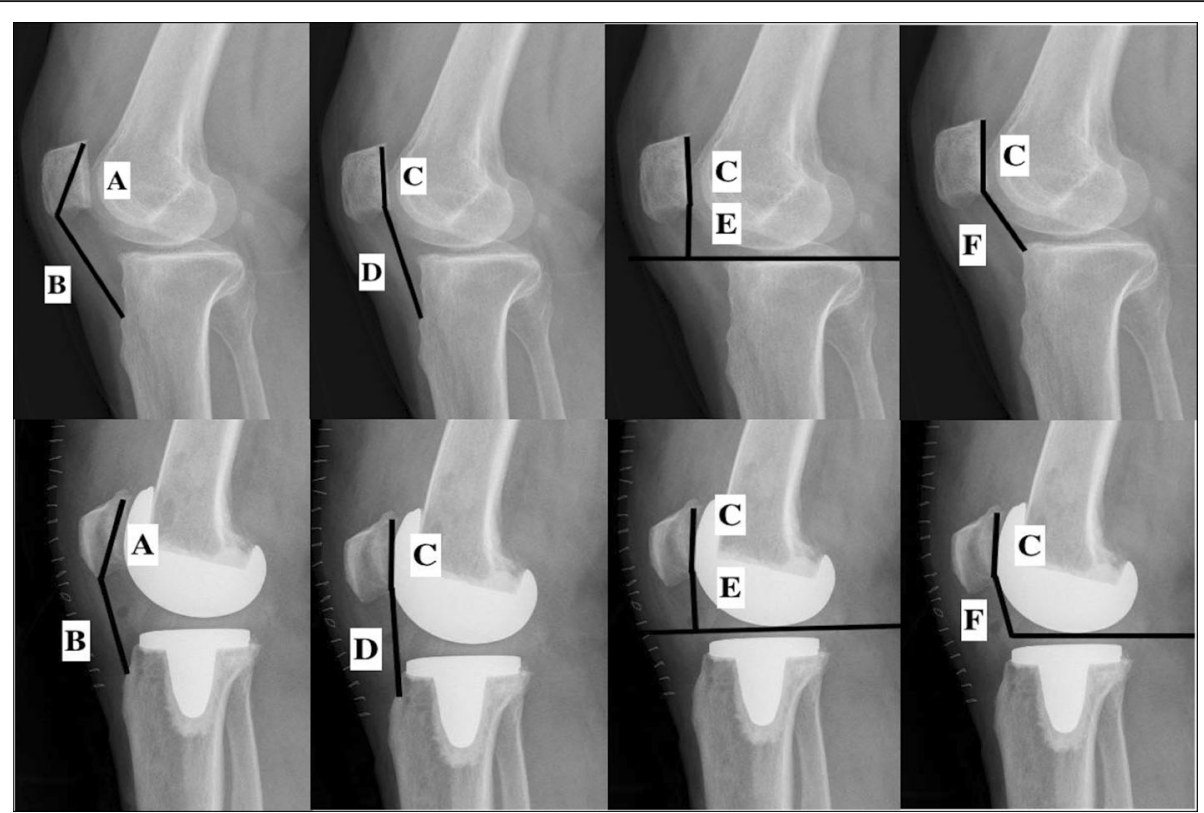

Fig. 1 Knee joint measurements. A: diagonal length of patella. B: length of patellar tendon. C: length of patella articular surface. D: distance from patellar articular surface to tibial tuberosity. E: perpendicular height from the plateau line of the tibia to the lower end of the patellar articular surface. F: distance between patellar articular surface and anterior border of the tibial plateau. IS index $=B / A, M I S$ index $=D / C, B P$ index $=E / C$, $C D$ index $=F / C$

decreased after TKA, reflecting joint elevation. The IS index (preoperative: $1.07 \pm 0.19$, postoperative: $1.07 \pm 0.17$; $P=1$ ) changed little after TKA because the patellar tendon was shortened when the diagonal length of the patella was reduced during patellar reshaping (see Table 2).

\section{Discussion}

Patellar tendon shortening and joint line elevation are two main causes of PB. Shortening of the patellar tendon is related to the reconstruction of the anterior cruciate ligament [18, 22, 23], patellar fracture [24], high tibial and distal femoral osteotomies $[25,26]$, and other factors. Although the standard medial parapatellar approach can provide a good operative field through patellar eversion [27-29], there is a risk of injuring the patellar tendon during the operation or even stripping the tendon from the tibial tubercle [30-32]. Additionally, the decreased strength of the quadriceps femoris muscle may cause contracture of the patellar tendon [32]. Floren et al. [17] reported that $37 \%$ of patients had patellar tendon shortening of $>5 \%$ through the standard medial parapatellar approach and that $14 \%$ of patients had shortening of $>10 \%$. Koshino et al. [15] reported that $65 \%$ of patients had patellar tendon shortening of $>10 \%$, while

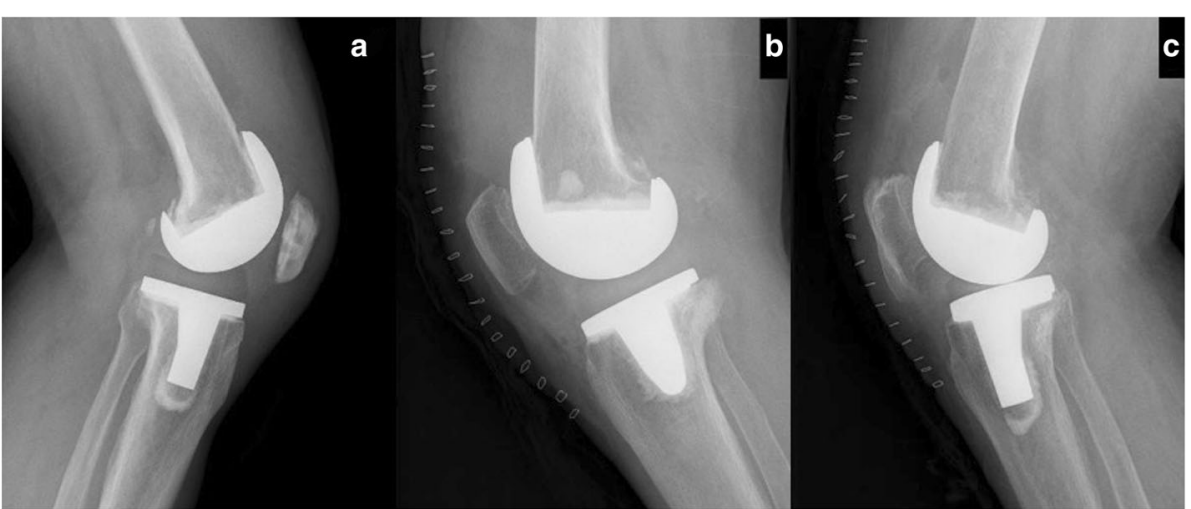

Fig. 2 Patients with postoperative TPB, PPB, and Cyrano patella. a A 63-year-old man with postoperative TPB. IS $=0.65, \mathrm{MIS}=0.75, \mathrm{BP}=0.22$, and $\mathrm{CD}=0.30$. b A 77-year-old man with postoperative PPB. IS $=0.90, \mathrm{MIS}=1.25, \mathrm{BP}=0.54$, and $\mathrm{CD}=0.56$. c A 75-year-old woman with postoperative Cyrano patella. IS $=0.54, \mathrm{MIS}=1.28, \mathrm{BP}=0.58$, and $\mathrm{CD}=0.59$ 
Table 1 Number of positive and negative cases of all indices before and after total knee arthroplasty

\begin{tabular}{cll}
\hline Index & Preoperation & Postoperation \\
\hline IS (+) & 20 & 21 \\
MIS (+) & 11 & 18 \\
MIS (-) & 9 & 5 \\
IS (-) & 236 & 235 \\
MIS (+) & 4 & 5 \\
MIS (-) & 232 & 230 \\
MIS (+)/(-) & $15 / 241$ & $23 / 233$ \\
BP (+) & 8 & 39 \\
CD (+) & 6 & 31 \\
CD (-) & 2 & 8 \\
BP (-) & 248 & 217 \\
CD (+) & 2 & 5 \\
CD (-) & 246 & 212 \\
CD (+)/(-) & $8 / 248$ & $36 / 220$
\end{tabular}

IS Insall-Salvati, MIS modified Insall-Salvati, BP Blackburne-Peel, $C D$ Caton-Deschamps, IS (+) number of cases of positive IS index, IS (-) number of cases of negative IS index, MIS (+) number of cases of positive MIS index, MIS (-) number of cases of negative MIS index, $B P(+)$ number of cases of positive $B P$ index, $B P(-)$ number of cases of negative $B P$ index, $C D(+)$ number of cases of positive $C D$ index, $C D(-)$ number of cases of negative $C D$ index

Weale et al. [16] reported a rate of 34\%. Joint line elevation may be the result of excessive soft tissue release, excessive distal femoral osteotomy [33, 34], insufficient tibial plateau osteotomy [35], and a large insert thickness [2]. Joint line elevation caused by TKA is not uncommon; the incidence can reach $40 \%$ after primary TKA [13, 36-38]. Kawamura and Bourne [39] reported a mean joint line elevation of $3.5 \mathrm{~mm}$, Sabbioni et al. [40] reported $4.0 \mathrm{~mm}$, and Scuderi and Insall [41] reported $5.6 \mathrm{~mm}$.

When patellar tendon shortening exceeds $10 \%$, a negative correlation exists between the shortening and ROM of the knee and between the patellar position and knee function [33]. Joint line elevation can change the knee kinematics $[35,37]$ and result in distal shift of the patella, increasing both the pressure on the patella when the knee is flexed and the tension on the soft tissue [42, 43]. Joint line elevation after TKA is likely to result in anterior knee pain, decreased ROM, decreased knee function, and patella component impingement on the tibia or polyethylene

Table 2 Comparison of all indices before and after total knee arthroplasty

\begin{tabular}{llll}
\hline & Preoperation & Postoperation & $P$ \\
\hline IS index & $1.07 \pm 0.19$ & $1.07 \pm 0.17$ & 1 \\
MIS index & $1.61 \pm 0.22$ & $1.51 \pm 0.21$ & $<0.0001$ \\
BP index & $0.86 \pm 0.16$ & $0.74 \pm 0.14$ & $<0.0001$ \\
CD index & $0.93 \pm 0.15$ & $0.78 \pm 0.12$ & $<0.0001$
\end{tabular}

IS Insall-Salvati, MIS modified Insall-Salvati, BP Blackburne-Peel, CD Caton-Deschamps
[21, 33, 35]. Estupinan et al. [44] claimed that a shift in the joint line itself induces wear of the polyethylene, and Rand [43] suggested that greater proximal joint line shifts correspond to more distal patellar movement compared with the preoperative position. Partington et al. [45] reported that joint line elevation of $>8 \mathrm{~mm}$ yields inferior clinical results, and Porteous et al. [46] found that joint line elevation of $>5 \mathrm{~mm}$ worsened clinical outcomes. Emodi et al. [47] suggested that even mild joint line elevation of $<2 \mathrm{~mm}$ alters the transmission of myogenic strength in the quadriceps and increases patellofemoral pressure forces.

In the present study, the preoperative incidence of TPB was higher than PPB, indicating that patellar tendon shortening was the main reason. The MIS, BP, and $\mathrm{CD}$ indices decreased after the operation, indicating that the incidence of PB increased and that both patellar tendon shortening and joint line elevation played a role in this change. Although the IS index changed little after the operation, the incidence of PB diagnosed by the IS index increased when false-positive cases were excluded. Thus, the incidence of PB increased after TKA. In addition, the incidence of TPB increased from 9.4\% (24 cases) to $10.2 \%$ (26 cases) after TKA, while the incidence of PPB increased from $0.8 \%$ ( 2 cases) to $9.0 \%$ (23 cases). Because the increase of PPB was higher, the PB caused by TKA was more strongly associated with joint line elevation. Restoration of the proper joint line is therefore a critical factor for successful TKA, indicating that evaluation of the patellar height before and after TKA is important.

Performing X-ray examinations of the knee joint is necessary before and after TKA. The IS, MIS, BP, and $\mathrm{CD}$ indices are simple and commonly used measures with which to evaluate the patellar height, and they can be measured on a roentgenogram. Although measurements using computed tomography or magnetic resonance imaging may be more accurate, we prefer using roentgenography because it has the following advantages. The IS and MIS indices are used to evaluate the length of the patellar tendon, and both of these indices use the tibial tuberosity as a landmark. The BP and CD indices are used to evaluate the height of the joint line, and both of these indices use the tibial plateau as a landmark. The IS and MIS indices can detect TPB, but they cannot detect PPB caused by joint line elevation. The BP and $\mathrm{CD}$ indices are important measures with which to detect $\mathrm{PPB}$ because they synthetically consider the length of the patellar tendon and the height of the joint line. The combination of these two indices will help to more comprehensively evaluate $\mathrm{PB}$ and provide a basis for detecting TPB or PPB.

The indices employed to evaluate the patellar height involve a ratio between two measurements. One measures the patellar length, and the other measures the 
distance between the patella and the tibia. A ratio can compensate for variations in patients' height, while an absolute length cannot. Although numerous indices are available to evaluate the patellar height, no single index is perfect. The IS index is more likely to be influenced by the shape of the patella. The incidence of PB will be overestimated in patients with a Cyrano patella (i.e., patella with a long nonarticulating inferior pole) (see Fig. 2c) [48]. The MIS index is not influenced by the length of the inferior pole, and its use will decrease the rate of false-positive cases. The consistency between the IS and MIS indices was moderate preoperatively but better postoperatively because false-positive cases of the IS index were present preoperatively and eliminated through patella reshaping. Thus, the MIS index is more suitable for evaluation of the length of the patellar tendon preoperatively, and both the IS and MIS indices are suitable for evaluation of the length of the patellar tendon postoperatively. The BP index requires a line to be drawn along the tibial plateau, which is inconvenient to do on a roentgenogram, and the overlap of the medial and lateral plateaus must be perfect for accurate placement of the line. However, placement of a perfect line on the roentgenogram rarely occurs in every case. Although the preoperative consistency between the BP and $C D$ indices was relatively good in our study, the CD index is a better choice for evaluation of the distance between the distal pole of the patella and the tibial plateau. Locating the anterior border of the insert is more difficult postoperatively because it is invisible on the roentgenogram. Therefore, postoperatively, the BP index is a better choice for evaluation of the distance between the distal pole of the patella and the tibial plateau; even the postoperative consistency was good.

Seo et al. [49] proposed an effective method with which to prevent joint line elevation through application of an additional metal block to reduce excess resection of the distal femur, successfully decreasing the incidence of PPB from 92 to $11 \%$. However, they chose patients who had $\mathrm{a} \leq 15-\mathrm{mm}$ perpendicular height from the plateau line of the tibia to the lower end of the patellar articular surface. As mentioned above, an absolute length cannot compensate for the variations in patients' height. Determination of the optimal range of a proper index is therefore needed to prevent PB. In this study, we provide evidence for choosing the proper index with which to evaluate the patellar height and changing different indices after TKA, thus establishing a basis for finding such a range.

This study had several limitations. The radiological measurements obtained by roentgenography may have been influenced by the presence of severe osteophytes or inaccurate patient positioning (e.g., deviation from the $30^{\circ}$ flexed position or image overlays). Because the positive rate of $\mathrm{PB}$ was low, the number of positive cases was small in our study. This study simply demonstrated the radiologic properties before and after TKA without clinical data. Further investigations of clinical outcomes among patients with TPB, PPB, or a normal patella are needed.

\section{Conclusion}

In conclusion, the IS and MIS indices can be used to detect TPB. The MIS index is more suitable for preoperative evaluation of the length of the patellar tendon. Although both the IS index and the MIS index are suitable for evaluating the length of the patellar tendon postoperatively, the MIS index is a better choice. Along with the IS and MIS indices, the BP and CD indices are important in detecting PPB. Although both the BP and $\mathrm{CD}$ indices are suitable for evaluating the distance between the distal pole of the patella and the tibial plateau, the $\mathrm{CD}$ index is a better choice preoperatively and the $\mathrm{BP}$ index is a better choice postoperatively. $\mathrm{PB}$ caused by TKA is more strongly associated with joint line elevation, which means that the $\mathrm{CD}$ and $\mathrm{BP}$ indices are of greater importance for the diagnosis of $\mathrm{PB}$ after TKA.

\section{Abbreviations \\ BP: Blackburne-Peel; CD: Caton-Deschamps; IS: Insall-Salvati; MIS: Modified Insall-Salvati; PB: Patella baja; PPB: Pseudo-patella baja; ROM: Range of motion; TKA: Total knee arthroplasty; TPB: True patella baja}

\section{Acknowledgements}

We thank Angela Morben, DVM, ELS, from Liwen Bianji, Edanz Editing China (www.liwenbianji.cn/ac), for editing the English text of a draft of this manuscript.

\section{Funding}

The study was supported by the Chinese Medicine Research Program of Zhejiang Province (CN) (2017ZA017).

\section{Availability of data and materials}

The datasets used and/or analyzed during the current study are available from the corresponding author on reasonable request.

\section{Authors' contributions}

BX was responsible for the data interpretation and analysis, literature search, and manuscript writing. WX was responsible for the surgical procedures and manuscript writing. DL and HS interpreted and analyzed the index

measuring data. XX was responsible for the data interpretation and analysis and literature search. DG was responsible for the literature search and manuscript writing. All authors read and approved the final manuscript.

\section{Ethics approval and consent to participate}

The study protocol was approved by the institutional review board (IRB) of Tongde Hospital of Zhejiang Province. Written informed consent was prospectively obtained from all patients before surgery.

\section{Consent for publication}

The IRB waived the requirement to obtain consent because this was a purely retrospective study that followed the standard of care.

\section{Competing interests}

The authors declare that they have no competing interests.

\section{Publisher's Note}

Springer Nature remains neutral with regard to jurisdictional claims in published maps and institutional affiliations. 
Received: 2 September 2017 Accepted: 28 November 2017 Published online: 12 December 2017

\section{References}

1. van der List JP, Sheng DL, Kleeblad LJ, et al. Outcomes of cementless unicompartmental and total knee arthroplasty: a systematic review. Knee. 2017:24:497-507.

2. Davies GS, van Duren B, Shorthose M, et al. Changes in patella tendon length over 5 years after different types of knee arthroplasty. Knee Surg Sports Traumatol Arthrosc. 2016;24:3029-35

3. Kazemi SM, Daftari Besheli L, Eajazi A, et al. Pseudo-patella baja after total knee arthroplasty. Medical science monitor: international medical journal of experimental and clinical research. 2011;17:CR292-6.

4. Jawhar A, Sohoni S, Shah V, et al. Alteration of the patellar height following total knee arthroplasty. Arch Orthop Trauma Surg. 2014;134:91-7.

5. Prudhon $\mathrm{J}$, , Caton $\mathrm{JH}$, Aslanian $\mathrm{T}$, et al. How is patella height modified after total knee arthroplasty? Int Orthop. 2017; doi:10.1007/s00264-017-3539-6.

6. Pinsornsak P, Naratrikun K, Chumchuen S. The effect of infrapatellar fat pad excision on complications after minimally invasive TKA: a randomized controlled trial. Clin Orthop Relat Res. 2014:472:695-701.

7. Gwyn R, Kotwal RS, Holt MD, et al. Complete excision of the infrapatellar fat pad is associated with patellar tendon shortening after primary total knee arthroplasty. European journal of orthopaedic surgery \& traumatology. 2016;26:545-9.

8. Vives-Barquiel MA, Torrents A, Lozano L, et al. Proximalize osteotomy of tibial tuberosity (POTT) as a treatment for stiffness secondary to patella baja in total knee arthroplasty (TKA). Arch Orthop Trauma Surg. 2015;135:1445-51.

9. Meneghini RM, Ritter MA, Pierson $J L$, et al. The effect of the Insall-Salvati ratio on outcome after total knee arthroplasty. J Arthroplast. 2006;21:116-20.

10. Ee G, Pang HN, Chong HC, et al. Computer navigation is a useful intra-operative tool for joint line measurement in total knee arthroplasty. Knee. 2013;20:256-62.

11. Insall J, Salvati E. Patella position in the normal knee joint. Radiology. 1971:101:101-4

12. Grelsamer RP, Meadows S. The modified Insall-Salvati ratio for assessment of patellar height. Clin Orthop Relat Res. 1992;282:170-6.

13. Blackburne JS, Peel TE. A new method of measuring patellar height. The Journal of bone and joint surgery British volume. 1977:59:241-2.

14. Caton J, Deschamps G, Chambat P, et al. Patella Infera. Apropos of 128 cases. Revue de chirurgie orthopedique et reparatrice de l'appareil moteur. 1982;68:317-25.

15. Koshino T, Ejima M, Okamoto R, et al. Gradual low riding of the patella during postoperative course after total knee arthroplasty in osteoarthritis and rheumatoid arthritis. J Arthroplast. 1990:5:323-7.

16. Weale AE, Murray DW, Newman JH, et al. The length of the patellar tendon after unicompartmental and total knee replacement. The Journal of bone and joint surgery British volume. 1999;81:790-5.

17. Floren M, Davis J, Peterson MG, et al. A mini-midvastus capsular approach with patellar displacement decreases the prevalence of patella baja. J Arthroplast. 2007;22:51-7

18. Dandy DJ, Desai SS. Patellar tendon length after anterior cruciate ligament reconstruction. The Journal of bone and joint surgery British volume. 1994:76:198-9.

19. Jawhar A, Hutter K, Scharf HP. Are joint line changes after primary navigated total knee arthroplasty predictable? Journal of orthopaedic science: official journal of the Japanese Orthopaedic Association. 2015;20:93-100.

20. Wegrzyn J, Parratte S, Coleman-Wood K, et al. The John Insall award: no benefit of minimally invasive TKA on gait and strength outcomes: a randomized controlled trial. Clin Orthop Relat Res. 2013:471:46-55.

21. Caton JH, Prudhon JL, Aslanian T, et al. Patellar height assessment in total knee arthroplasty: a new method. Int Orthop. 2016;40:2527-31.

22. Shaffer BS, Tibone JE. Patellar tendon length change after anterior cruciate ligament reconstruction using the midthird patellar tendon. Am J Sports Med. 1993:21:449-54.

23. Tria AJ Jr, Alicea JA, Cody RP. Patella baja in anterior cruciate ligament reconstruction of the knee. Clin Orthop Relat Res. 1994;299:229-34.

24. Mariani PP, Del Signore S, Perugia L. Early development of patella infera after knee fractures. Knee Surg Sports Traumatol Arthrosc. 1994;2:166-9.

25. Okamoto R, Koshino T, Morii T. Shortening of patellar ligament and patella baja with improvement of quadriceps muscle strength after high tibia osteotomy. Bulletin. 1993;53:21-4.
26. Scuderi GR, Windsor RE, Insall JN. Observations on patellar height after proximal tibial osteotomy. J Bone Joint Surg Am. 1989:71:245-8.

27. Engh GA, Holt BT, Parks NL. A midvastus muscle-splitting approach for total knee arthroplasty. J Arthroplast. 1997;12:322-31.

28. Hofmann AA, Plaster RL, Murdock LE. Subvastus (Southern) approach for primary total knee arthroplasty. Clin Orthop Relat Res. 1991;269:70-7.

29. Zan P, Wu Z, Yu X, et al. The effect of patella eversion on clinical outcome measures in simultaneous bilateral total knee arthroplasty: a prospective randomized controlled trial. J Arthroplast. 2016;31:637-40.

30. Paulos LE, Rosenberg TD, Drawbert J, et al. Infrapatellar contracture syndrome. An unrecognized cause of knee stiffness with patella entrapment and patella infera. Am J Sports Med. 1987;15:331-41.

31. Wojtys EM, Oakes B, Lindenfeld TN, et al. Patella Infera syndrome: an analysis of the patellar tendon pathology. Instr Course Lect. 1997;46:241-50.

32. Noyes FR, Wojtys EM, Marshall MT. The early diagnosis and treatment of developmental patella infera syndrome. Clin Orthop Relat Res. 1991;265:241-52.

33. Chonko DJ, Lombardi AV Jr, Berend KR. Patella baja and total knee arthroplasty (TKA): etiology, diagnosis, and management. Surgical technology international. 2004;12:231-8.

34. Cope MR, O'Brien BS, Nanu AM. The influence of the posterior cruciate ligament in the maintenance of joint line in primary total knee arthroplasty: a radiologic study. J Arthroplast. 2002:17:206-8.

35. Figgie HE 3rd, Goldberg VM, Heiple KG, et al. The influence of tibial-patellofemora location on function of the knee in patients with the posterior stabilized condylar knee prosthesis. The Journal of bone and joint surgery American. 1986;68:1035-40.

36. Laskin RS. Joint line position restoration during revision total knee replacement. Clin Orthop Relat Res. 2002:404:169-71.

37. Bellemans J. Restoring the joint line in revision TKA: does it matter? Knee. 2004;11:3-5.

38. Yoshii I, Whiteside LA, White SE, et al. Influence of prosthetic joint line position on knee kinematics and patellar position. J Arthroplast. 1991;6:169-77.

39. Kawamura $\mathrm{H}$, Bourne RB. Factors affecting range of flexion after total knee arthroplasty. J Orthop Sci. 2001;6: 248-52.

40. Sabbioni G, Rani N, Del Piccolo N, et al. Gap balancing versus measured resection technique using a mobile-bearing prosthesis in computer-assisted surgery. Musculoskelet Surg. 2011:95:25-30.

41. Scuderi GR, Insall JN. Total knee arthroplasty. Current clinical perspectives. Clin Orthop Relat Res. 1992;276:26-32.

42. Goldberg VM, Figgie HE 3rd, Figgie MP. Technical considerations in total knee surgery. Management of patella problems. The Orthopedic clinics of North America. 1989;20:189-99.

43. Rand JA. Patellar resurfacing in total knee arthroplasty. Clin Orthop Relat Res. 1990:260:110-7.

44. Estupinan JA, Bartel DL, Wright TM. Residual stresses in ultra-high molecular weight polyethylene loaded cyclically by a rigid moving indenter in nonconforming geometries. J Orthop Res. 1998;16:80-8.

45. Partington PF, Sawhney J, Rorabeck $\mathrm{CH}$, et al. Joint line restoration after revision total knee arthroplasty. Clin Orthop Relat Res. 1999;367:165-71.

46. Porteous AJ, Hassaballa MA, Newman JH. Does the joint line matter in revision total knee replacement? The Journal of bone and joint surgery British volume. 2008;90:879-84

47. Emodi GJ, Callaghan JJ, Pedersen DR, et al. Posterior cruciate ligament function following total knee arthroplasty: the effect of joint line elevation. The lowa orthopaedic journal. 1999:19:82-92.

48. Grelsamer RP, Proctor CS, Bazos AN. Evaluation of patellar shape in the sagittal plane. A clinical analysis. Am J Sports Med. 1994:22:61-6.

49. Seo JG, Moon YW, Kim SM, et al. Prevention of pseudo-patella baja during tota knee arthroplasty. Knee Surg Sports Traumatol Arthrosc. 2015;23:3601-6. 